\title{
Glutamine + glutamate level predicts the magnitude of microstructural organization in the gray matter in the healthy elderly
}

\author{
Tomokazu Motegi, ${ }^{1}$ Kosuke Narita, ${ }^{2}$ Kazuyuki Fujihara, ${ }^{1}$ Masato Kasagi, ${ }^{1}$ \\ Yusuke Suzuki, ${ }^{1}$ Minami Tagawa, ${ }^{1}$ Koichi Ujita, ${ }^{3}$ Jamie Near, ${ }^{4}$ and Masato Fukuda \\ ${ }^{1}$ Department of Psychiatry and Neuroscience, Gunma University Graduate School of Medicine, 3-39-22 Showa-machi, Maebashi, Gunma 371-8511, fapan \\ ${ }^{2}$ Department of Psychiatry, Graduate School of Medical Science, Kyoto Prefectural University of Medicine, Kawaramachi-Hirokoji, Kamigyo-ku, Kyoto \\ 602-8566, fapan \\ ${ }^{3}$ Department of Diagnostic Radiology and Nuclear Medicine, Gunma University Graduate School of Medicine, Maebashi, Gunma 371-8511, fapan \\ ${ }^{4}$ Douglas Mental Health University Institute and Department of Psychiatry, McGill University, Montreal, QC, Canada
}

Background: Diffusion tensor imaging (DTI), which is a technique for measuring the degree and direction of movement of water molecules in tissue, has been widely used to noninvasively assess white matter (WM) or gray matter (GM) microstructures in vivo. Mean diffusivity (MD), which is the average diffusion across all directions, has been considered as a marker of WM tract degeneration or extracellular space enlargement in GM. Recent lines of evidence suggest that cortical MD can better identify early-stage Alzheimer's disease than structural morphometric parameters in magnetic resonance imaging. However, knowledge of the relationships between cortical MD and other biological factors in the same cortical region, e.g. metabolites, is still limited.

Methods: Thirty-three healthy elderly individuals [aged 50-77 years (mean, 63.8 47.4 years); 11 males and 22 females] were enrolled. We estimated the associations between cortical MD and neurotransmitter levels. Specifically, we measured levels of $\gamma$-aminobutyric acid (GABA) and glutamate + glutamine (Glx), which are inhibitory and excitatory neurotransmitters, respectively, in medial prefrontal cortex (mPFC) and posterior cingulate cortex (PCC) using MEGA-PRESS magnetic resonance spectroscopy, and we measured regional cortical MD using DTI.

Results: Cortical MD was significantly negatively associated with Glx levels in both mPFC and PCC. No significant association was observed between cortical MD and GABA levels in either GM region.

Conclusion: Our findings suggest that degeneration of microstructural organization in GM, as determined on the basis of cortical MD measured by DTI, is accompanied by the decline of Glx metabolism within the same GM region.

Key words: diffusion tensor imaging, mean diffusivity, magnetic resonance spectroscopy, glutamine + glutamate (Glx), $\gamma$-aminobutyric acid (GABA)

\section{Introduction}

Over the last 30 years, various methods of magnetic resonance imaging (MRI) have been developed and used to assess brain alterations associated with normal aging and aged-related neurodegenerative diseases, such as mild cognitive impairment and

Correspondence should be addressed to: Tomokazu Motegi, Department of Psychiatry and Neuroscience, Gunma University Graduate School of Medicine, 3-39-22 Showa-machi, Maebashi, Gunma 371-8511, Japan. Phone: +81 27220 8185; Fax: +81 27220 8187. Email address: motegi@atr.jp (T. Motegi). Received 08 Mar 2019; revision requested 24 May 2019; revised version received 22 Jul 2019; accepted 19 Aug 2019. First published online 03 October 2019.
Alzheimer's disease (Elman et al., 2017). Diffusion tensor imaging (DTI), a reliable MRI technique for measuring the degree and direction of movement of water molecules in brain tissue, has been widely used to noninvasively assess white matter (WM) or gray matter (GM) microstructures in vivo (Le Bihan et al., 1986). Fractional anisotropy (FA), which is thought to be an index of WM integrity calculated by DTI, can reflect the state of neural fibers on the basis of the density, diameter, or coherence of axons (Le Bihan et al., 2001). Mean diffusivity (MD), which is the average magnitude of diffusion across all directions, has been considered as a marker of WM tract degeneration or 
extracellular space enlargement in GM (Elman et al., 2017; Neil et al., 2002).

Many previous DTI studies have revealed altered FA and MD even in normal aging (Abe et al., 2008; Benedetti et al., 2006; Garcia-Lazaro et al., 2016), age-related neurodegenerative diseases, such as mild cognitive impairment and Alzheimer's disease (Nesteruk et al., 2016; Nishioka et al., 2015), demyelinating diseases, such as multiple sclerosis (de Kouchkovsky et al., 2016), and neuropsychiatric diseases, such as depression and schizophrenia (Jiang et al., 2017; Singh et al., 2016). Most of these studies have focused on WM or deep GM such as the hippocampus, because these brain regions have high directionality in water diffusion (Manna et al., 2015; Ziyan and Westin, 2008), whereas water diffusion in the cerebral cortex has an isotropic direction at the level of conventional DTI resolution (Elman et al., 2017).

On the other hand, recent lines of DTI evidence have shown that altered cortical MD may reflect the magnitude of microstructural organization in GM, that is, patients with mild cognitive impairment or Alzheimer's disease have been reported to show increased cortical MD, compared with healthy controls in several GM regions including the posterior cingulate cortex (PCC), entorhinal cortex, amygdala, parahippocampal gyrus, middle temporal gyrus, superior and middle frontal gyri and bilateral supramarginal gyri (Lin et al., 2016; Ray et al., 2006; Rose et al., 2008; Weston et al., 2015). Furthermore, cortical MD begins to increase more generally in middle age (Ni et al., 2010). Noteworthy, a recent twin study showed that cortical MD is associated with genetic factors, distinct from cortical thickness or MD in WM (Elman et al., 2017), suggesting that cortical MD measurement by DTI may have greater potential use for assessing degeneration of microstructural organization in the GM associated with normal aging or age-related diseases than morphometric GM volume estimation in T1-weighted imaging. However, knowledge of relationships between $\mathrm{MD}$ and metabolite levels in the same cortical regions is still limited.

In this study of healthy elderly people without dementia, we examined the associations between cortical MD and the levels of $\gamma$-aminobutyric acid (GABA) and glutamine + glutamate (Glx), which are inhibitory and excitatory neurotransmitters, respectively, in the medial prefrontal cortex (mPFC) and PCC by DTI and magnetic resonance spectroscopy (MRS) using MEGA-PRESS (Bauer et al., 2013; Jocham et al., 2012). Decreased levels of GABA and Glx in several cortical areas including $\mathrm{mPFC}$ and PCC have been reported to be associated with aging (Goryawala et al., 2016; Grachev et al., 2001) and to be found in patients with mild cognitive
Table 1. Demographic characteristics of study subjects

\begin{tabular}{lr}
\hline MALE : FEMALE $(n)$ & $11: 22$ \\
Age (y) & $63.8 \pm 7.4$ \\
Education (y) & $14.0 \pm 1.9$ \\
MOCA (point) & $26.3 \pm 2.6$ \\
JART - predicted full-scale & $112.2 \pm 7.6$ \\
IQ (point) & \\
Cambridge Neuropsychological & \\
$\quad$ Test Automated Battery & \\
Spatial recognition memory & $81.9 \pm 10.8$ \\
$\quad$ (percent correct) & \\
$\quad$ Rapid Visual Information & \\
$\quad$ Processing (A') & \\
\hline $\begin{array}{l}\text { MoCA, the Montreal Cognitive Assessment; JART, Japanese } \\
\text { version of National Adult Reading Test; A', signal detection }\end{array}$ \\
measures of accuracy, Mean \pm SD.
\end{tabular}

impairment and Alzheimer's disease (Antuono et al., 2001; Huang et al., 2016; Riese et al., 2015), that is, $\mathrm{mPFC}$ or PCC has been recognized as one of the most important brain areas associated with memory loss or cognitive decline in elderly people. In addition, decreased Glx levels in certain GM regions have been suggested to be associated with neuronal dysfunction and loss in that GM region (Segovia et al., 2001). We hypothesized that cortical $\mathrm{MD}$ in $\mathrm{MPFC}$ and PCC correlates negatively with the levels of GABA and Glx in the same volume of interest (VOI).

\section{Methods}

\section{Subjects}

Thirty-three individuals [aged 50-77 years (mean, $63.8 \pm 7.4$ years); 11 males and 22 females] were enrolled in this study (Table 1 ) on the basis of the following exclusion criteria: history of dementia, neurological or psychiatric illness, diabetes mellitus, chronic alcoholism, smoking, and obesity with a body mass index above 25. All subjects were right-handed, as assessed using the Edinburgh Handedness Inventory (Oldfield, 1971), and provided their written informed consent. The study protocol was approved by the Ethics Committee of Gunma University.

\section{Psychological measurements}

The Montreal Cognitive Assessment (MoCA) was administered to all subjects for the screening of cognitive function (Ihara et al., 2013; Nasreddine et al., 2005); although several cut-off points have been proposed for MoCA, we used scores $\geq 20$ to exclude subjects with dementia and cognitive impairment, in accordance with a previous report 
A

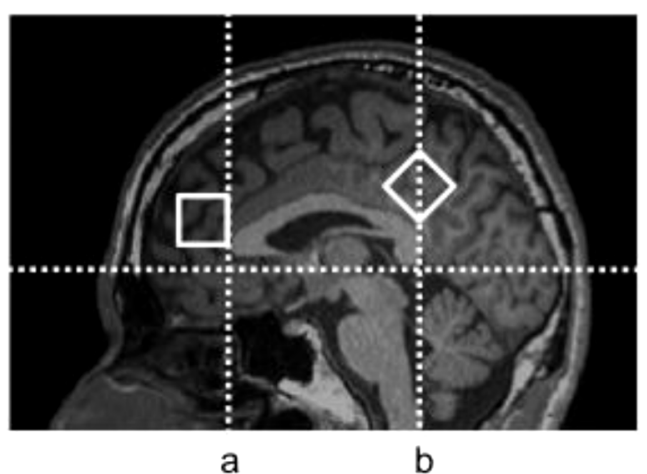

B

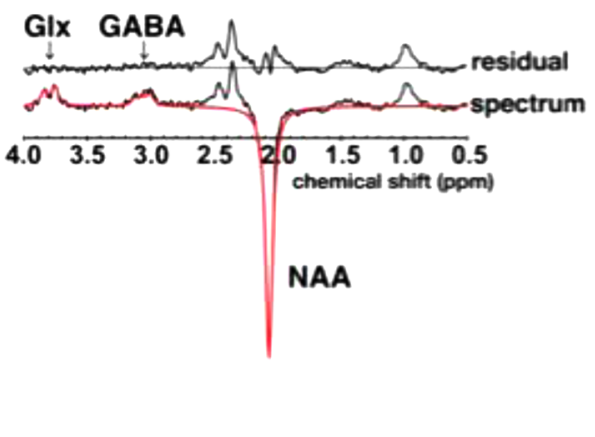

Figure 1. Magnetic resonance spectroscopy (MRS) using MEGA-PRESS. The volumes of interest $\left(30 \times 20 \times 20 \mathrm{~mm}^{3}\right)$ in $\mathrm{MRS}$, which were located on mPFC and PCC, are shown in A; "line $a$ " is set exactly on the rostral margin of the corpus callosum as the perpendicular axis to the anterior commissure-posterior commissure (AC-PC) line. "line $b$ " is drawn as the perpendicular axis through the anterior commissure to the AC-PC line. The edited spectrum (i.e. black line) and fitted curve (i.e. red line), which were obtained for the signal quantification of GABA, $\mathrm{Glx}$, and $\mathrm{Cr}$ levels, are shown in $\mathrm{B}$.

(Waldron-Perrine and Axelrod, 2012). The Japanese version of the National Adult Reading Test was then conducted to estimate premorbid IQs (Matsuoka et al., 2006; Nelson, 1982). The Cambridge neuropsychological test automated battery [CANTAB (Morris et al., 1987); Cambridge Cognition Ltd., Cambridge, United Kingdom] was also administered to each subject of this study and consisted of the following: the Spatial Recognition Memory (SRM) test of visual spatial memory in a two-choice forced discrimination paradigm and the Rapid Visual Information Processing (RVIP) test, which is a visual continuous performance task using digits instead of letters.

\section{Acquisition of MRS and DTI and T1-weighted anatomical imaging data}

MRS ACQUISITION

Edited GABA and Glx MR spectra were acquired using the MEGA-PRESS sequence (Mescher et al., 1998) with the following acquisition parameters: $\mathrm{TR}=2400$ $\mathrm{ms}$; $\mathrm{TE}=68 \mathrm{~ms}$; number of averages $=512$ for the $\mathrm{mPFC}$ and 256 for PCC. In both regions, the prescribed MRS VOIs was $30 \times 20 \times 20 \mathrm{~mm}^{3}$. Based on the chemical shift difference between the 3 ppm GABA resonance and the $3.75 \mathrm{ppm}$ Glx resonance, the chemical shift displacement between GABA and Glx in the direction of the excitation pulse (bandwidth = $3708 \mathrm{~Hz}$, selective in the left-right direction), was $0.75 \mathrm{~mm}$. In the directions of refocusing pulses (bandwidth $=1106 \mathrm{~Hz}$, selective in the anteriorposterior direction and in the superior-inferior direction), the chemical shift displacement between GABA and Glx was $1.67 \mathrm{~mm}$. The VOI in $\mathrm{mPFC}$ was defined as follows. After drawing "line a" exactly on the rostral margin of the corpus callosum as the perpendicular axis to the anterior commissure-posterior commissure
(AC-PC) line (see Fig. 1A), the VOI in $\mathrm{mPFC}$ was set along "line a" and on the inferoposterior corner located at the rostral edge of the genu. The VOI in PCC was set above the superior surface of the corpus callosum together with the diagonal line of the VOI aligned along "line b," which is the perpendicular axis across the posterior edge of the splenium to the AC-PC line (see Fig. 1A).

We measured the full-width at half-maximum (FWHM) of $\mathrm{N}$-acetyl aspartate (NAA) peaks in the MEGA-PRESS spectra to determine the quality of shimming. The means \pm standard deviations (SDs) were $6.864 \pm 2.272 \mathrm{~Hz}$ in $\mathrm{mPFC}$ and 5.201 $\pm 2.203 \mathrm{~Hz}$ in PCC. We excluded the samples whose FWHM values of NAA peaks were large (i.e. FWHM $>$ mean \pm 2 SD). Although data from PCC were successfully collected from all the participants, those from $\mathrm{mPFC}$ of one participant were excluded from the analysis because the FWHM of the NAA peak was larger than the cut-off value. In addition, we evaluated motion artifacts by visual inspection of superimposed spectra for all the excitations during scanning using MEGA-PRESS. As a result, two samples obtained from $\mathrm{mPFC}$ were also excluded owing to motion artifacts.

DTI AND T1-WEIGHTED ANATOMICAL IMAGING DATA ACQUISITIONS

DTI data of the participants were acquired using a clinical 3.0 Tesla Prisma MRI scanner (Siemens, Erlangen, Germany) with a 12-channel head coil. The entire brain was scanned by echo-planar imaging with 30 noncolinear motion probing gradients at a b-value of $1000 \mathrm{sec} / \mathrm{mm}^{2}$ and $5 \mathrm{~T}_{2}$-weighted $\mathrm{b}=0$ images. The acquisition parameters for the DTI scan were as follows: $\mathrm{TE}=84 \mathrm{~ms}, \mathrm{TR}=6500 \mathrm{~ms}$, 50 axial slices, slice thickness $=3 \mathrm{~mm}$, field of 
view $=23 \times 23 \mathrm{~cm}^{2}$, and matrix size $=128 \times 128$ (i.e. voxel size $=1.8 \times 1.8 \times 3.0 \mathrm{~mm}^{3}$ ). To improve signal-to-noise ratio, the scanning was repeated twice [number of excitation $(\mathrm{NEX})=2$ ].

We also obtained high-resolution T1-weighted images for GM morphometry. MP-RAGE sequencing yielding 176 continuous slices of $1.0 \mathrm{~mm}$ thickness was carried out along the sagittal plane. The acquisition parameters were as follows: echo time $=2 \mathrm{~ms}$; repetition time $=2000 \mathrm{~ms}$; inversion time $=990 \mathrm{~ms}$; flip angle $=9^{\circ}$; field of view $=256 \mathrm{~mm}$; matrix size $=256 \times 256$; voxel size $=1 \times 1 \times 1 \mathrm{~mm}^{3}$.

\section{Processing and analysis of MRS and DTI data}

Measurement of Gaba and GLX Levels BY MRS

Prior to signal averaging, each scan was frequencyand phase-aligned by spectral registration (Near et al., 2015) using the FID-A toolkit (https:// github.com/cic-methods/fid-a) in MATLAB (MathWorks, Natick, MA, USA) to minimize the effects of frequency and phase drifts. Motion-corrupted averages were removed as described previously (Near et al., 2013; Simpson et al., 2017). Averaged difference spectra and sum spectra were then linebroadened using a 5-Hz Lorentzian filter, and zeroorder phase corrections were applied manually to ensure upright peaks. First-order phase corrections were also applied manually in some cases because the first point in the FID did not always correspond exactly to the top of the echo. Following preprocessing, GABA and Glx signals from the difference spectra and creatine $(\mathrm{Cr})$ signals from the sum spectra were quantified using the AMARES package provided in jMRUI software (Naressi et al., 2001; Vanhamme et al., 2001). GABA, Glx, and Cr were modeled as a triplet, a doublet, and a singlet of Lorentzian peaks, respectively. Because the editing efficacy for Glx has not been determined in the current sequence, Glx signals are shown in an arbitrary unit value.

Measurement of MD Within MRS VOIs Diffusion tensor data were converted into the NIfTI format using MRI Convert (http://lcni.uoregon.edu/ ;olinda/MRIConvert). The data were preprocessed using FMRIB Software Library (FSL) version 4.1.5 (http://www.fmrib.ox.ac.uk/fsl). This procedure included the following: (1) eddy current correction, (2) motion correction by registering all the diffusionweighted data to the $b=0$ images, which were corrected first, (3) brain extraction, (4) calculation of diffusion tensor and diagonalization, and (5) transformation to the MNI space. After these processes, the MD map in the MNI space was automatically constructed using FSL.

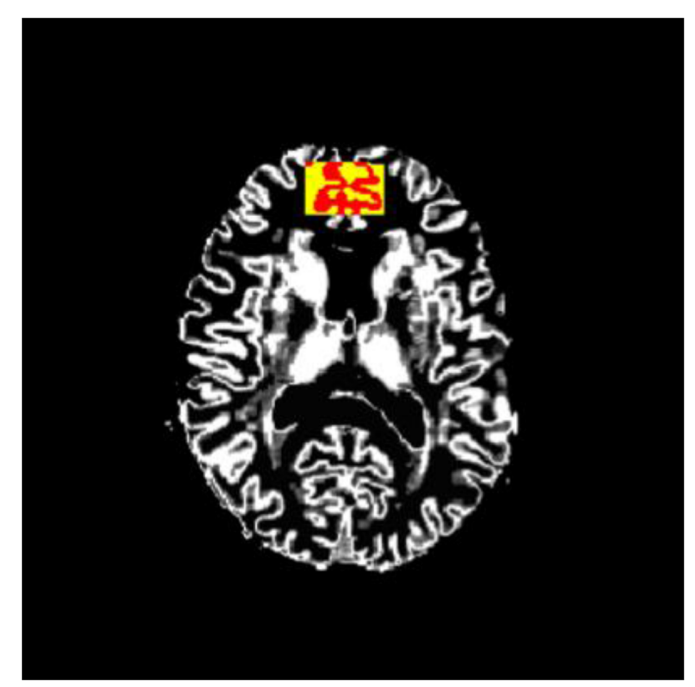

Figure 2. Binary mask of GM within $\mathrm{mPFC}$ VOI for measurement of $M D$. The figure shows a segmented GM image together with mPFC VOI in MRS (yellow) and a binary mask of GM within MPFC VOI in MRS (red) in the original space.

Then, the obtained T1-weighted anatomical images were segmented into GM, WM, and cerebrospinal fluid (CSF) using FAST (FMRIB's automated segmentation tool) (Zhang et al., 2001) in FSL software (available from http://fsl.fmrib.ox. ac.uk.proxy.bib.uottawa.ca/fsl/fslwiki/) (Jenkinson et al., 2012; Smith et al., 2004; Woolrich et al., 2009; Zhang et al., 2001) to calculate the relative volume of each tissue within MRS VOIs. After the binary GM masks within MRS VOIs were formed from segmented GM images and MRS VOIs (Fig. 2), Statistical Parametric Mapping (SPM; http://www.fil.ion.ucl.ac.uk/spm/) was used to coregister different spaces between the binary GM masks and the MD map in each subject. The threshold of GM mask was set to the default SPM parameter of 0.8. Because MD is markedly higher in CSF than in the brain tissue, the map of MDs lower than, $0.102 \times 10^{-2} \mathrm{~mm}^{2} / \mathrm{s}$ was generated to attenuate the CSF effect on MD measurement within MRS VOIs, in accordance with the previous method described by Albrecht et al. (2007), i.e. the above-mentioned cut-off values were set at 3 SDs above the mean measured tissue MD. Finally, we calculated mean $M D$ from nonzero voxels of the MD map within binary GM masks of MRS VOIs, i.e. MPFC and PCC.

\section{Statistical analysis}

The metabolite ratios are presented in the results section of this study, i.e. raw $\mathrm{GABA} / \mathrm{Cr}$ and $\mathrm{Glx} / \mathrm{Cr}$, and GM corrected GABA/Cr and $\mathrm{Glx} / \mathrm{Cr}$, which were divided by the relative volume of GM within each VOI in MRS in accordance with previous 


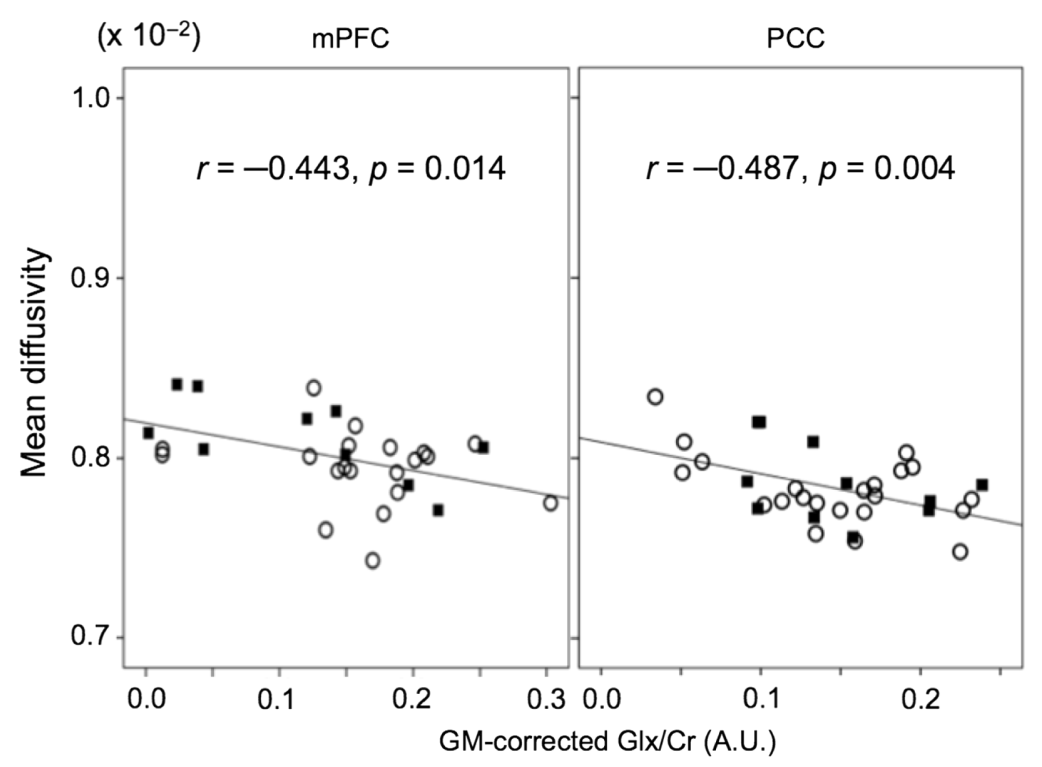

Figure 3. Scatter plots showing correlation between cortical MD and GM corrected Glx/Cr in mPFC and PCC. Squares indicate male subjects and circles indicate female subjects.

studies (Fujihara et al., 2015; Jocham et al., 2012). To assess the relationship among demographic characteristics, CANTAB scores, and metabolite ratios in MRS, Pearson's correlation test were performed. In addition, we also performed regression analysis, in accordance with a previous report (Yildiz et al., 2014). A p-value of $<0.05$ was set as statistically significant. Student's t-test was conducted to estimate gender differences in demographic characteristics. All the statistical analyses were carried out using SPSS version-24 (IBM Corporation).

\section{Results}

All the subjects showed MoCA scores $\geq 20$, and the predicted IQs were higher than 90 in all the participants (mean $\pm \mathrm{SD}=112.2 \pm 7.6$; see Table 1 ). Pearson's correlation test showed that in mPFC and PCC, NAA level, Cr level, raw GABA/Cr or Glx, or GM corrected GABA/Cr or Glx/Cr did not significantly correlate with age, education years, predicted IQ, MoCA score, or CANTAB test scores, i.e. SRM and RVIP scores. Furthermore, $\mathrm{MD}$ in $\mathrm{mPFC}$ and PCC did not significantly correlate with age, education years, predicted IQ, MoCA score, or the above-mentioned CANTAB test scores. The MD in $\mathrm{mPFC}$ or PCC did not correlate with $\mathrm{Cr}$ level in each VOI, respectively.

The raw Glx/Cr levels in mPFC and PCC were significantly negatively correlated with $\mathrm{MD}$ in each VOI $(r=-0.477, p=0.008$ and $r=-0.486$, $p=0.004$, respectively). The $\mathrm{GM}$ corrected $\mathrm{Glx} / \mathrm{Cr}$ levels in $\mathrm{mPFC}$ and PCC were also significantly negatively correlated with $\mathrm{MD}$ in each VOI $(r=-0.443, p=0.014$ and $r=-0.487, p=0.004$, respectively) (Fig. 3). No significant correlations were observed between $\mathrm{MD}$ in $\mathrm{mPFC}$ and Glx level in PCC and between MD in PCC and Glx level in $\mathrm{mPFC}$. Furthermore, raw or GM corrected GABA/Cr in $\mathrm{MPFC}$ and PCC did not significantly correlate with $\mathrm{MD}$ in either VOI.

In regression analysis, the raw $\mathrm{Glx} / \mathrm{Cr}$ in $\mathrm{mPFC}$ and PCC could predict $\mathrm{MD}$ in each VOI $\beta=-0.477$, $\mathrm{t}=-2.871, p=0.008$, and $\beta=-0.486, \mathrm{t}=-3.94$, $p=0.004)$. Also, the GM corrected $\mathrm{Glx} / \mathrm{Cr}$ in mPFC and PCC could predict MD in each VOI $(\beta=-0.443, \quad \mathrm{t}=-2.617, \quad p=0.014, \quad$ and $\beta=-0.487, \mathrm{t}=-3.102, p=0.004)$. To control further for possible effects of GM, WM and CSF fractions were used in separate regression models for raw $\mathrm{Glx} / \mathrm{Cr}$, as reported by Fujihara et al. (2015) and Yildiz et al. (2014). All these models revealed that neither GM, WM, nor CSF explained the further variance in the models (mPFC: $|\beta|<0.463,|t|<1.064$, $p>0.297$; and PCC: $|\beta|<0.131, \quad|t|<0.617$, $p>0.542$ ). These regression models showed that only raw $\mathrm{Glx} / \mathrm{Cr}$ has a predictive effect on $\mathrm{MD}$ in each VOI (mPFC: $\beta=-0.471, \mathrm{t}=-2.724$, $p=0.011 ;$ and PCC: $\beta=-0.478, \mathrm{t}=-2.908$, $p=0.007)$. Also, GM corrected $\mathrm{Glx} / \mathrm{Cr}$ has a predictive effect on $\mathrm{MD}$ in each VOI (mPFC: $\beta=-0.422$, $\mathrm{t}=-2.68, p=0.021 ;$ and PCC: $\beta=-0.477$, $\mathrm{t}=-2.975, p=0.006)$.

\section{Discussion}

The results of this study show that cortical MD is significantly negatively correlated with Glx levels in 
both $\mathrm{mPFC}$ and PCC in healthy elderly individuals. On the other hand, no significant association was found between GABA level and cortical $M D$ in both VOIs.

Glx level is very likely related to excitatory neurotransmission, because glutamate is considered to be the major component of the Glx signal (Bauer et al., 2013; Fujihara et al., 2015). The dominant pathway for glutamine production is via metabolism of neurotransmitter glutamate, so Glx represents the integrated metabolic and neurotransmitter functions of glutamate in the brain (Jahng et al., 2016; Yuksel and Ongur, 2010). The glutamate level in a certain GM region is suggested to be associated with neuronal dysfunction and loss in that GM region (Segovia et al., 2001). Thus, previous MRS studies showed that a decreased Glx level can be observed in neurodegenerative conditions, such as aging (Goryawala et al., 2016; Grachev et al., 2001), mild cognitive impairment and Alzheimer's disease (Antuono et al., 2001; Huang et al., 2016; Riese et al., 2015), in several GM regions including mPFC and PCC, which implies neuronal dysfunction and loss in these GM regions. Consistently, cognitive performance or cognitive symptoms have been reported to positively correlate with Glx levels in elderly people (Zahr et al., 2008) and patients with mild cognitive impairment (Nikolova et al., 2017) and Alzheimer's disease (Walecki et al., 2011). Then, an increased cortical MD, which has been observed in aging and the above-mentioned diseases (Lin et al., 2016; Ni et al., 2010; Ray et al., 2006; Weston et al., 2015), may reflect the decline in the magnitude of microstructural organization in the GM associated with aging and age-related diseases, i.e. a breakdown of cytoarchitectural barriers such as the cell membrane or a shift in the concentration of water between intra- and extracellular spaces (Elman et al., 2017; Neil et al., 2002; Sundgren et al., 2004; Van Camp et al., 2012). Considering the above-mentioned previous reports on Glx level and cortical MD, the significant negative association between cortical MD and Glx level in MPFC and PCC observed in this study suggests that age-related neuronal loss, which can be detected on the basis of cortical MD in DTI, leads to the decline of Glx metabolism within the above-mentioned GM regions.

In this study, no significant association was found between GABA levels and cortical MD in both $\mathrm{mPFC}$ and PCC. Similarly to Glx levels, previous MRS studies have shown that the GABA levels in $\mathrm{mPFC}$ and PCC are lower in healthy elderly people and patients with Alzheimer's disease and mild cognitive impairments than in controls (Bai et al., 2015; Riese et al., 2015). Moreover, cognitive dysfunction has been reported to be associated with decreased GABA levels in mild cognitive impairment and Alzheimer's disease (Porges et al., 2017). Although such observations suggest that not only Glx levels, but also GABA levels reflect age-related neuronal loss, this speculation is inconsistent with the findings of our study. There are some difficulties in explaining the different findings on Glx and GABA levels in this study, but an asymmetric degeneration of glutamatergic neurons and GABAergic neurons during aging might contribute to our results regarding GABA in this study, that is, terminals and synapses of glutamatergic neurons, not those of GABAergic neurons, are reported to be predominantly affected in early-stage Alzheimer's disease (Huang et al., 2016; Kashani et al., 2008; Proctor et al., 2010). Additional studies including age-related neurodegenerative disorders, i.e. mild cognitive impairment and Alzheimer's disease, should be carried out. Furthermore, the lack of significant GABA observations might have been due to the small sample size in this study.

A major limitation in this study is its small sample size, which may have influenced our statistical results. In addition, owing to the lack of data from subjects with age-related neurodegenerative disorders, i.e. mild cognitive impairment or Alzheimer's disease, there are some limitations in this study. Although some previous MRS studies have shown the significant associations between cognitive function test scores and the levels of several cortical metabolites, i.e. NAA, GABA or Glx (Jessen et al., 2013; Porges et al., 2017), we failed to find such significant associations, which may be due to the small number of subjects in this study. In addition, young subjects should also be enrolled. Further MRS studies with larger sample sizes including subjects of various ages and with age-related neurodegenerative disorders will reinforce our findings in the future.

In conclusion, the level of Glx, which reflects excitatory neurotransmission, was associated with cortical MD in $\mathrm{MPFC}$ and PCC in GM. These findings might support a hypothesis, i.e. neuronal loss detected on the basis of cortical MD in DTI can cause a decline of Glx metabolism in these GM regions in elderly people. The results of this study can lead to a better understanding of the association between microstructural organization and neuronal metabolism in GM during aging.

\section{Conflict of interest}

None. 


\section{Description of authors' roles}

T. Motegi collected and analyzing the data and wrote the paper. K. Narita was responsible for the statistical design of the study and for carrying out the statistical analysis. K. Fujihara, M. Kasagi, Y. Suzuki and M. Tagawa collected the data and assisted with analyzing the data. K. Ujita helped carrying the study out. J. Near contributed to MRI and MRS methods and assisted with writing the article. M. Fukuda supervised the data collection and assisted with writing the article.

\section{Acknowledgment}

This work was supported by JSPS KAKENHI JP Grant Numbers 23591695 and 15KK0295.

\section{References}

Abe, O. et al. (2008). Aging in the CNS: comparison of gray/ white matter volume and diffusion tensor data. Neurobiology of Aging, 29, 102-116.

Albrecht, J. et al. (2007). Voxel based analyses of diffusion tensor imaging in Fabry disease. Fournal of Neurology, Neurosurgery, and Psychiatry, 78, 964-969.

Antuono, P.G., Jones, J.L., Wang, Y. and Li, S.J., (2001). Decreased glutamate + glutamine in Alzheimer's disease detected in vivo with (1)H-MRS at 0.5 T. Neurology, 56, 737-742.

Bai, X. et al. (2015). Decreased gamma-aminobutyric acid levels in the parietal region of patients with Alzheimer's disease. Fournal of Magnetic Resonance Imaging, 41, 1326-1331.

Bauer, J. et al. (2013). Craving in alcohol-dependent patients after detoxification is related to glutamatergic dysfunction in the nucleus accumbens and the anterior cingulate cortex. Neuropsychopharmacology, 38, 1401-1408.

Benedetti, B. et al. (2006). Influence of aging on brain gray and white matter changes assessed by conventional, MT, and DT MRI. Neurology, 66, 535-539.

de Kouchkovsky, I., Fieremans, E., Fleysher, L., Herbert, J., Grossman, R.I. and Inglese, M., (2016). Quantification of normal-appearing white matter tract integrity in multiple sclerosis: a diffusion kurtosis imaging study. Fournal of Neurology, 263, 1146-1155.

Elman, J.A. et al. (2017). Genetic and environmental influences on cortical mean diffusivity. Neuroimage, 146, 90-99.

Fujihara, K. et al. (2015). Relationship of gammaaminobutyric acid and glutamate + glutamine concentrations in the perigenual anterior cingulate cortex with performance of Cambridge Gambling Task. Neuroimage, 109, 102-108.

Garcia-Lazaro, H.G., Becerra-Laparra, I., Cortez-Conradis, D. and Roldan-Valadez, E., (2016). Global fractional anisotropy and mean diffusivity together with segmented brain volumes assemble a predictive discriminant model for young and elderly healthy brains: a pilot study at 3T. Functional Neurology, 31, 39-46.

Goryawala, M.Z., Sheriff, S. and Maudsley, A.A., (2016). Regional distributions of brain glutamate and glutamine in normal subjects. NMR in Biomedicine, 29, 1108-1116.

Grachev, I.D., Swarnkar, A., Szeverenyi, N.M., Ramachandran, T.S. and Apkarian, A.V., (2001). Aging alters the multichemical networking profile of the human brain: an in vivo (1)H-MRS study of young versus middleaged subjects. Fournal of Neurochemistry, 77, 292-303.

Huang, D., Liu, D., Yin, J., Qian, T., Shrestha, S. and Ni, H., (2016). Glutamate-glutamine and GABA in brain of normal aged and patients with cognitive impairment. European Radiology, 27, 2698-2705.

Ihara, M., Okamoto, Y. and Takahashi, R., (2013). Suitability of the Montreal cognitive assessment versus the mini-mental state examination in detecting vascular cognitive impairment. Fournal of Stroke and Cerebrovascular Diseases, 22, 737-741.

Jahng, G.H. et al. (2016). Glutamine and glutamate complex, as measured by functional magnetic resonance spectroscopy, alters during face-name association task in patients with mild cognitive impairment and Alzheimer's disease. Fournal of Alzheimer's Disease, 52, 145-159.

Jenkinson, M., Beckmann, C.F., Behrens, T.E., Woolrich, M.W. and Smith, S.M., (2012). FSL. Neuroimage, 62, 782-790.

Jessen, F. et al. (2013). $N$-acetylaspartylglutamate (NAAG) and $\mathrm{N}$-acetylaspartate (NAA) in patients with schizophrenia. Schizophrenia Bulletin, 39, 197-205.

Jiang, J. et al. (2017). Microstructural brain abnormalities in medication-free patients with major depressive disorder: a systematic review and meta-analysis of diffusion tensor imaging. Fournal of Psychiatry \& Neuroscience, 42, 150-163.

Jocham, G., Hunt, L.T., Near, J. and Behrens, T.E., (2012). A mechanism for value-guided choice based on the excitation-inhibition balance in prefrontal cortex. Nature Neuroscience, 15, 960-961.

Kashani, A., Lepicard, E., Poirel, O., Videau, C., David, J.P., Fallet-Bianco, C., Simon, A., Delacourte, A., Giros, B., Epelbaum, J., Betancur, C. and El Mestikawy, S., (2008). Loss of VGLUT1 and VGLUT2 in the prefrontal cortex is correlated with cognitive decline in Alzheimer disease. Neurobiology of Aging, 29, 1619-1630.

Le Bihan, D., Breton, E., Lallemand, D., Grenier, P., Cabanis, E. and Laval-Jeantet, M., (1986). MR imaging of intravoxel incoherent motions: application to diffusion and perfusion in neurologic disorders. Radiology, 161, 401-407.

Le Bihan, D. et al. (2001). Diffusion tensor imaging: concepts and applications. Fournal of Magnetic Resonance Imaging, 13, 534-546.

Lin, S.H. et al. (2016). Increased Water Diffusion in the Parcellated Cortical Regions from the Patients with Amnestic Mild Cognitive Impairment and Alzheimer's Disease. Frontiers in Aging Neuroscience, 8, 325.

Manna, A., Piras, F., Caltagirone, C., Bossu, P., Sensi, S.L. and Spalletta, G., (2015). Left hippocampusamygdala complex macro- and microstructural variation is associated with BDNF plasma levels in healthy elderly individuals. Brain and Behavior, 5, e00334. 
Matsuoka, K., Uno, M., Kasai, K., Koyama, K. and Kim, Y., (2006). Estimation of premorbid IQ in individuals with Alzheimer's disease using Japanese ideographic script (Kanji) compound words: Japanese version of National Adult Reading Test. Psychiatry and Clinical Neurosciences, 60, 332-339.

Mescher, M., Merkle, H., Kirsch, J., Garwood, M. and Gruetter, R., (1998). Simultaneous in vivo spectral editing and water suppression. NMR in Biomedicine, 11, 266-272.

Morris, R.G., Evenden, J.L., Sahakian, B.J. and Robbins, T.W., (1987). Computer-aided assessment of dementia: comparative studies of neuropsychological deficits in Alzheimer-type dementia and Parkinson's disease. In: S. Stahl, S. Iversen, E. Goodman (Eds.), Cognitive Neurochemistry (pp. 21-36). Oxford: Oxford University Press.

Naressi, A., Couturier, C., Castang, I., de Beer, R. and Graveron-Demilly, D., (2001). Java-based graphical user interface for MRUI, a software package for quantitation of in vivo/medical magnetic resonance spectroscopy signals. Computers in Biology and Medicine, 31, 269-286.

Nasreddine, Z.S. et al. (2005). The Montreal Cognitive Assessment, MoCA: a brief screening tool for mild cognitive impairment. Fournal of the American Geriatrics Society, 53, 695-699.

Near, J. et al. (2013). Unedited in vivo detection and quantification of gamma-aminobutyric acid in the occipital cortex using short-TE MRS at $3 \mathrm{~T}$. NMR in Biomedicine, 26, 1353-1362.

Near, J., Edden, R., Evans, C.J., Paquin, R., Harris, A. and Jezzard, P., (2015). Frequency and phase drift correction of magnetic resonance spectroscopy data by spectral registration in the time domain. Magnetic Resonance in Medicine, 73, 44-50.

Neil, J., Miller, J., Mukherjee, P. and Huppi, P.S., (2002). Diffusion tensor imaging of normal and injured developing human brain - a technical review. NMR in Biomedicine, 15, 543-552.

Nelson, H.E., (1982). National Adult Reading Test (NART): For the assessment of premorbid intelligence in patients with dementia: Test manual. NFER-Nelson. Windsor, United Kingdom.

Nesteruk, T., Nesteruk, M., Styczynska, M., Barcikowska-Kotowicz, M. and Walecki, J., (2016). Radiological evaluation of strategic structures in patients with mild cognitive impairment and early Alzheimer's disease. Polish fournal of Radiology, 81, 288-294.

Ni, J.M., Chen, S., Liu, J.J., Huang, G., Shen, T.Z. and Chen, X.R., (2010). Regional diffusion changes of cerebral grey matter during normal aging-a fluid-inversion prepared diffusion imaging study. European fournal of Radiology, 75, 134-138.

Nikolova, S., Stark, S.M. and Stark, C.E.L., (2017). 3T hippocampal glutamate-glutamine complex reflects verbal memory decline in aging. Neurobiology of Aging, 54, 103-111.

Nishioka, C., Poh, C. and Sun, S.W., (2015). Diffusion tensor imaging reveals visual pathway damage in patients with mild cognitive impairment and Alzheimer's disease. Fournal of Alzheimer's Disease, 45, 97-107.

Oldfield, R.C., (1971). The assessment and analysis of handedness: the Edinburgh inventory. Neuropsychologia, 9, 97-113.
Porges, E.C. et al. (2017). Frontal gamma-aminobutyric acid concentrations are associated with cognitive performance in older adults. Biological Psychiatry: Cognitive Neuroscience and Neuroimaging, 2, 38-44.

Proctor, D.T., Coulson, E.J. and Dodd, P.R., (2010). Reduction in post-synaptic scaffolding PSD-95 and SAP102 protein levels in the Alzheimer inferior temporal cortex is correlated with disease pathology. Fournal of Alzheimer's Disease, 21, 795-811.

Ray, K.M. et al. (2006). Mild cognitive impairment: apparent diffusion coefficient in regional gray matter and white matter structures. Radiology, 241, 197-205.

Riese, F. et al. (2015). Posterior cingulate gammaaminobutyric acid and glutamate/glutamine are reduced in amnestic mild cognitive impairment and are unrelated to amyloid deposition and apolipoprotein E genotype. Neurobiology of Aging, 36, 53-59.

Rose, S.E., Janke, A.L. and Chalk, J.B., (2008). Gray and white matter changes in Alzheimer's disease: a diffusion tensor imaging study. Fournal of Magnetic Resonance Imaging, 27, 20-26.

Segovia, G., Porras, A., Del Arco, A. and Mora, F., (2001). Glutamatergic neurotransmission in aging: a critical perspective. Mechanisms of Ageing and Development, 122, $1-29$.

Simpson, R., Devenyi, G.A., Jezzard, P., Hennessy, T.J. and Near, J., (2017). Advanced processing and simulation of MRS data using the FID-Appliance (FID-A) - An open source, MATLAB based toolkit. Magnetic Resonance in Medicine, 77, 23-33.

Singh, S. et al. (2016). Microstructural abnormalities of uncinate fasciculus as a function of impaired cognition in schizophrenia: A DTI study. Fournal of Biosciences, 41, 419-426.

Smith, S.M. et al. (2004). Advances in functional and structural MR image analysis and implementation as FSL. Neuroimage, 23, Suppl 1, S208-219.

Sundgren, P.C., Dong, Q., Gomez-Hassan, D., Mukherji, S.K., Maly, P. and Welsh, R., (2004). Diffusion tensor imaging of the brain: review of clinical applications. Neuroradiology, 46, 339-350.

Van Camp, N. et al. (2012). A complementary diffusion tensor imaging (DTI)-histological study in a model of Huntington's disease. Neurobiology of Aging, 33, 945-959.

Vanhamme, L., Sundin, T., Hecke, P.V. and Huffel, S.V., (2001). MR spectroscopy quantitation: a review of timedomain methods. NMR in Biomedicine, 14, 233-246.

Waldron-Perrine, B. and Axelrod, B.N., (2012). Determining an appropriate cutting score for indication of impairment on the Montreal Cognitive Assessment. International fournal of Geriatric Psychiatry, 27, 1189-1194.

Walecki, J., Barcikowska, M., Cwikla, J.B. and Gabryelewicz, T., (2011). $N$-acetylaspartate, choline, myoinositol, glutamine and glutamate (glx) concentration changes in proton MR spectroscopy (1H MRS) in patients with mild cognitive impairment (MCI). Medical Science Monitor, 17, Mt105-111.

Weston, P.S., Simpson, I.J., Ryan, N.S., Ourselin, S. and Fox, N.C., (2015). Diffusion imaging changes in grey matter in Alzheimer's disease: a potential marker of early neurodegeneration. Alzheimer's Research \& Therapy, 7, 47. 
Woolrich, M.W. et al. (2009). Bayesian analysis of neuroimaging data in FSL. Neuroimage, 45, S173-186.

Yildiz, A. et al. (2014). Feeling safe in the plane: neural mechanisms underlying superior action control in airplane pilot trainees-a combined EEG/MRS study. Human Brain Mapping, 35, 5040-5051.

Yuksel, C. and Ongur, D., (2010). Magnetic resonance spectroscopy studies of glutamate-related abnormalities in mood disorders. Biological Psychiatry, 68, 785-794.

Zahr, N.M., Mayer, D., Pfefferbaum, A. and Sullivan, E.V., (2008). Low striatal glutamate levels underlie cognitive decline in the elderly: evidence from in vivo molecular spectroscopy. Cerebral Cortex, 18, 2241-2250.

Zhang, Y., Brady, M, and Smith, S., (2001). Segmentation of brain MR images through a hidden Markov random field model and the expectationmaximization algorithm. IEEE Transactions on Medical Imaging, 20, 45-57.

Ziyan, U. and Westin, C.F., (2008). Joint segmentation of thalamic nuclei from a population of diffusion tensor MR images. Medical Image Computing and Computer-Assisted Intervention, 11, 279-286. 